\title{
Évaluation des obstacles empêchant l'accès aux denrées chez les habitants de Cobourg (Ontario) en situation d'insécurité alimentaire
}

\author{
S. Tsang, M. Sc. S., Dt. P. (1); A. M. Holt, M. Sc. S. (2); E. Azevedo, M. Sc., Dt. P. (1)
}

\section{Résumé}

Introduction : Les personnes à faible revenu sont les plus vulnérables à l'insécurité alimentaire; elles sont ainsi nombreuses à se tourner vers les programmes communautaires ou caritatifs d'aide alimentaire afin de bénéficier de denrées gratuites ou à faible coût. Cette évaluation des besoins vise à mieux connaître les obstacles à l'accès aux programmes d'aide alimentaire, les solutions envisageables pour améliorer l'accès aux denrées, les obstacles à la consommation de fruits et de légumes et les solutions envisageables pour augmenter celle-ci chez les personnes en situation d'insécurité alimentaire vivant à Cobourg (Ontario).

Méthodologie : Nous avons réalisé auprès de clients des programmes d’aide alimentaire des entrevues individuelles structurées comportant surtout des questions ouvertes.

Résultats : Les clients des programmes d'aide alimentaire ont indiqué que les obstacles à l'utilisation des services étaient le manque de moyens de transport et le fait que ces programmes offraient des quantités d'aliments insuffisantes ou avaient des heures d'ouverture peu pratiques. Ils ont également fait état d'un manque de fruits et de légumes à la maison et du fait que leur revenu était un obstacle à la consommation de ces aliments. Ils ont suggéré la mise en place d'un programme d'achat en grande quantité de fruits et légumes produits localement et le recours aux jardins communautaires, ce qui contribuerait à améliorer leur consommation de ces aliments.

Discussion : Grâce à une collaboration avec les partenaires communautaires, la plupart des obstacles cités pourraient être levés et les solutions mentionnées mises en place, ce qui aiderait les personnes à faible revenu à progresser vers la sécurité alimentaire.

Mots clés : nutrition, population à faible revenu, pauvreté, aliments sains, accessibilité, fruits, légumes, insécurité alimentaire, Ontario

\section{Introduction}

Le lien entre faible revenu et santé a été établi à maintes reprises : les personnes dont les conditions socio-économiques sont les plus défavorables sont vulnérables aux maladies chroniques, notamment les cardiopathies, le diabète, les maladies respiratoires chroniques et le cancer, et risquent de mourir prématurément $^{1-13}$. Selon plusieurs études, en situation d'insécurité alimentaire ${ }^{20-21}$. Ce sont les personnes à faible revenu qui sont les plus vulnérables à l'insécurité alimentaire : familles monoparentales, prestataires de l'aide sociale, locataires, sans abri, travailleurs pauvres, chômeurs, travailleurs non qualifiés et semi qualifiés, personnes ayant des besoins en alphabétisation, personnes atteintes de maladie mentale ou de toxicomanie, parents d'adolescents et personnes ayant une déficience ${ }^{22}$.

En Ontario, 47,2 \% des ménages gagnant moins de 10000 \$ avant impôt souffrent d'insécurité alimentaire, contre seulement $1,8 \%$ des ménages de la catégorie de revenu supérieure, 5,2 \% de ceux de la catégorie moyenne supérieure et $14,4 \%$ de ceux de la catégorie moyenne ${ }^{23}$. Les personnes en situation d'insécurité alimentaire se tournent soit vers les programmes d'alimentation offerts dans la collectivité, comme les jardins communautaires ou les cuisines collectives, soit vers les programmes offerts par des organismes caritatifs, par exemple les banques alimentaires, ou encore utilisent ces deux sources pour recevoir des aliments gratuits ou à faible coût, ce qui diminue un peu leur fardeau financier.

L'objet de cette évaluation des besoins est de recueillir, auprès des résidents de Cobourg (Ontario) en situation d'insécurité alimentaire, de l'information sur les éléments suivants : les obstacles à l'accès aux programmes alimentaires proposés par la collectivité ou par des organismes caritatifs; les solutions envisageables pour améliorer l'accès aux denrées; les obstacles à la consommation de légumes et de fruits et enfin les solutions 
envisageables pour augmenter la consommation de légumes et de fruits.

\section{Contexte}

Cobourg est situé en Ontario, à environ 110 kilomètres à l'est de Toronto. C'est le plus important centre urbain du Comté de Northumberland, majoritairement rural. En 2006, sa population s'élevait à 18210 habitants, la majorité ayant plus de 25 ans $^{24}$. On y comptait 5235 familles, dont $18 \%$ monoparentales ${ }^{24}$. Le taux de chômage y était de 6,7 \% (il est de 6,4\% pour l'Ontario) ${ }^{24}$. Environ $7 \%$ de la population de Cobourg avait un faible revenu avant impôt ${ }^{24}$.

Cobourg est desservi par un réseau de transport en commun qui compte deux lignes d'autobus à itinéraire fixe. On y trouve quatre magasins de grandes chaînes d'alimentation, dont deux sont des magasins à prix réduit. La ville compte une banque alimentaire, un programme de repas du midi gratuits, un jardin communautaire et quelques organisations communautaires caritatives gérant les dons de nourriture distribués gratuitement aux personnes en situation d'insécurité alimentaire. La banque alimentaire est ouverte le mercredi et le vendredi matin pendant trois heures, et chaque client peut bénéficier de ses services deux fois par mois. Le programme de repas du midi gratuits est offert tous les jours de la semaine pendant une heure à toute personne dans le besoin. Les organisations communautaires caritatives offrent les services liés aux dons de nourriture durant leurs heures normales d'ouverture.

\section{Méthodologie}

Nous avons utilisé un échantillon de convenance pour recueillir de l'information auprès d'adultes ayant utilisé au moins une fois les services de banques alimentaires ou de programmes de counseling.

Les répondants potentiels ont été recrutés grâce à deux organisations non gouvernementales locales qui gèrent des programmes caritatifs d'aide alimentaire, mais dont le mandat principal n'est pas de fournir de la nourriture. Notre choix s'est arrêté sur ces programmes parce qu'ils sont offerts dans des quartiers sûrs et accueillants, ce qui a facilité le recrutement des répondants et la réalisation des entrevues. De plus, ces deux organisations s'adressent non seulement aux personnes susceptibles d'utiliser tous les programmes caritatifs d'aide alimentaire disponibles, mais également à un éventail plus large de personnes en situation d'insécurité alimentaire. Quatre membres d'un comité local sur la sécurité alimentaire se sont portés volontaires pour suivre une formation afin d'effectuer les entrevues. On a posé des affiches dans les établissements choisis pour faire connaître notre étude d'évaluation des besoins. Nous avons utilisé deux stratégies d'échantillonnage : sur l'un des sites, deux des enquêteurs ayant reçu une formation approchaient les clients du programme d'aide alimentaire en lisant un texte standard; sur l'autre site, une personne connaissant bon nombre des clients du programme a facilité le recrutement des répondants. Dans les deux cas, la participation était volontaire. On expliquait aux participants potentiels l'objet de l'évaluation des besoins et on les assurait de la confidentialité du processus; ils donnaient leur consentement verbalement. Chaque participant était ensuite invité à s'installer dans un endroit calme ou dans un local indépendant, où deux intervieweurs procédaient à l'entrevue à partir du guide d'entrevue. Au total, 35 entrevues ont été réalisées, et les répondants ayant complété le processus ont reçu une lettre d'information décrivant notre évaluation des besoins et les modalités de leur consentement.

Le guide d'entrevue contenait des questions structurées et ouvertes et plusieurs questions fermées. Il avait été examiné par un membre du comité local de sécurité alimentaire et du personnel de l'unité de santé et avait fait l'objet d'un essai pilote auprès d'un échantillon de clients d'une banque alimentaire dans une autre municipalité. Les questions portaient sur les obstacles à l'utilisation des programmes d'aide alimentaire et les solutions envisageables pour améliorer l'accès à de tels programmes; sur les obstacles à la consommation de fruits et de légumes et les solutions envisageables pour augmenter la consommation de ces aliments, et enfin sur ce que signifiait, pour les répondants, le fait d'avoir suffisamment de nourriture. Les intervieweurs consignaient les mots clés, les expressions utilisées et les explications formulées par les répondants, notant en même temps leur langage corporel pour détailler le contexte de chaque réponse. Ils confirmaient la fiabilité des données consignées en répétant périodiquement la réponse, afin de s'assurer d'avoir bien compris et interprété ce qu'avait dit le répondant.

À la fin de chaque journée, tous les intervieweurs faisaient un bilan des réponses écrites et de ce qu'ils avaient pensé, ressenti et perçu à propos de chaque entrevue.

Le protocole d'évaluation des besoins a été jugé conforme aux normes éthiques du Bureau de santé du district d'Haliburton, Kawartha et Pine Ridge.

\section{Analyse des données}

Nous avons analysé qualitativement toutes les réponses aux questions ouvertes ainsi que toutes les notes prises lors des entrevues pour les questions ouvertes et fermées grâce à la méthode de l'analyse du cadre ${ }^{25}$. Il s'agit d'une méthode qualitative qui se prête particulièrement bien aux études caractérisées par des questions précises, un échéancier limité, un échantillon de convenance et des objectifs a priori, du type des obstacles et des possibilités évalués dans le cadre de notre étude ${ }^{26}$. On a séparé les comptages et les calculs de pourcentages pour chaque question fermée et les données personnelles. Les données ont été analysées durant le processus de collecte d'information et à l'issue de celui ci, ce qui a permis au chercheur principal de déterminer à quel moment le point de saturation des données avait été atteint.

Les réponses à toutes les questions de l'entrevue et les notes correspondantes ont été saisies dans un tableau en format Microsoft Word en utilisant la méthode de La Pelle ${ }^{27}$. Les données ont été codées au moyen d'un cadre thématique élaboré antérieurement à partir des objectifs de l'évaluation des besoins ${ }^{25}$. Les expressions indicatrices d'obstacles ou de possibilités ont été identifiées par des couleurs et des polices de caractère différentes, puis un document distinct a été créé pour les regrouper ${ }^{28}$. Elles ont été triées en différentes catégories : 1) obstacles et possibilités, selon le numéro de la question correspondante et la référence aux programmes d'aide alimentaire en général; 2) raison du recours aux programmes d'aide alimentaire plus 
TABLEAU 1

Caractéristiques des répondants $(\mathrm{N}=35)$ qui ont pris part à l'entrevue d'évaluation des besoins sur les obstacles à l'accès aux programmes alimentaires et à la consommation de fruits et de légumes

\begin{tabular}{|c|c|c|}
\hline Groupe & $\begin{array}{l}\text { Nombre de répondants } \\
\qquad \mathrm{n}\end{array}$ & $\begin{array}{l}\text { Pourcentage de répondants } \\
\%\end{array}$ \\
\hline \multicolumn{3}{|l|}{ Sexe } \\
\hline Femmes & 31 & 89 \\
\hline Hommes & 4 & 11 \\
\hline \multicolumn{3}{|l|}{ Âge } \\
\hline $18-29$ ans & 5 & 14 \\
\hline $30-39$ ans & 12 & 34 \\
\hline $40-49$ ans & 12 & 34 \\
\hline $50-59$ ans & 5 & 14 \\
\hline $60-69$ ans & 1 & 3 \\
\hline 70 ans et plus & 0 & 0 \\
\hline \multicolumn{3}{|l|}{ Lieu de résidence } \\
\hline Cobourg & 30 & 86 \\
\hline Extérieur de Cobourg & 5 & 14 \\
\hline \multicolumn{3}{|l|}{ Approche utilisée pour le recrutement } \\
\hline Les intervieweurs ont sollicité directement les répondants potentiels & 9 & 26 \\
\hline $\begin{array}{l}\text { Les intervieweurs ont été présentés aux répondants potentiels par une personne } \\
\text { qui les connaissait }\end{array}$ & 26 & 74 \\
\hline \multicolumn{3}{|l|}{ Enfants dans le ménage ${ }^{a}$} \\
\hline Aucun & 12 & 34 \\
\hline Enfants de 12 ans et moins & 18 & 51 \\
\hline Enfants de 13 à 18 ans & 10 & 29 \\
\hline \multicolumn{3}{|l|}{ Nombre de personnes travaillant dans le ménage } \\
\hline 0 & 13 & 37 \\
\hline 1 & 15 & 43 \\
\hline 2 & 5 & 14 \\
\hline 3 & 2 & 6 \\
\hline \multicolumn{3}{|l|}{ Source des revenus du ménage $\mathrm{e}^{\mathrm{b}}$} \\
\hline Ontario au travail & 10 & 29 \\
\hline Programme ontarien de soutien aux personnes handicapées & 11 & 31 \\
\hline $\begin{array}{l}\text { Supplément de revenu de l'Ontario pour les familles travailleuses ayant des frais } \\
\text { de garde d'enfants }\end{array}$ & 5 & 14 \\
\hline Programme de prestations d'invalidité du Régime de pensions du Canada (PPIRPC) & 0 & 0 \\
\hline Régime de pensions du Canada (RPC) & 3 & 9 \\
\hline Sécurité de la vieillesse & 2 & 6 \\
\hline Régime d'aide financière aux étudiantes et étudiants de l'Ontario & 0 & 0 \\
\hline Emploi régulier & 22 & 63 \\
\hline Assurance-emploi (AE) & 22 & 63 \\
\hline $\begin{array}{l}\text { Indemnités de la Commission de la sécurité professionnelle et de l'assurance contre } \\
\text { les accidents du travail (CSPAAT) }\end{array}$ & 0 & 0 \\
\hline Autre & 2 & 6 \\
\hline Aucun revenu & 0 & 0 \\
\hline
\end{tabular}

${ }^{a}$ Certains ménages comptent des enfants appartenant aux deux groupes d'âge (moins de 12 ans, 13 à 18 ans); dans ces cas-là, la somme des pourcentages est supérieure à 100 .

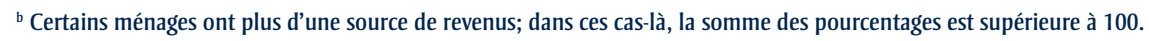


d'une fois par mois; 3) consommation de légumes et de fruits. On a ensuite analysé ces regroupements afin d'y déceler des sous-thèmes.

La question du texte standard de l'entrevue "Que signifie pour vous "avoir assez de nourriture”? » ne s’intégrait pas au cadre a priori. Elle permettait plutôt d'établir le contexte et la signification de l'insécurité alimentaire telle qu'elle était vécue localement par les individus et les ménages. Pour cette question, des thèmes ont donc été élaborés en fonction des données sans passer par un cadre a priori.

Deux chercheurs ont analysé toutes les données brutes de manière indépendante à partir du même cadre, et un troisième a réexaminé leur analyse. Les trois chercheurs ont discuté de toutes les divergences relevées afin de parvenir à un consensus au sujet des catégories.

\section{Résultats}

Le tableau 1 indique les caractéristiques des répondants et la composition de leur ménage.

Sur les 35 répondants qui ont pris part à l'entrevue, $43 \%$ ont déclaré que, s'il n'y avait pas de restriction, ils souhaiteraient profiter des programmes d'aide alimentaire une fois par semaine (données non indiquées). Tous les répondants ont affirmé qu'ils aimaient manger des légumes, $97 \%$ ont déclaré aimer les fruits. Ils n'ont été que $23 \%$ à dire qu'ils pouvaient manger autant de fruits et de légumes qu'ils le désiraient.

\section{Les obstacles à l'utilisation des programmes d'aide alimentaire}

L’obstacle le plus fréquemment mentionné par les répondants était le transport, puisque 14 répondants (40\%) n'avaient pas de moyen de transport leur permettant de se rendre au lieu où le programme était offert (pas de véhicule ou de possibilité de se faire conduire), ou encore avaient de la difficulté à rentrer chez eux à pied tout en portant de gros cartons remplis de denrées. Les réserves de certaines denrées distribuées par les banques alimentaires, comme le lait, les pâtes et le beurre d'arachide, s'épuisent rapidement, et 6 répondants (17\%) ont mentionné que les quantités d'aliments qu'ils recevaient étaient insuffisantes pour eux-mêmes et leur ménage. Au sujet de l'approvisionnement en denrées des banques alimentaires en général, une femme d’âge moyen vivant seule s'est exclamée : «C'est la nourriture - ils manquent de nourriture tout le temps ». Une autre répondante, mère de deux jeunes enfants et de deux adolescents, a affirmé : "La banque alimentaire ne distribue pas assez de nourriture ". Dix répondants $(29 \%)$ se sont plaints du fait que les programmes d'aide alimentaire n’ouvraient pas leur établissement pendant assez longtemps durant la journée et le mois, et que les heures d'ouverture n'étaient pas compatibles avec leur horaire personnel. Une femme a expliqué le fonctionnement actuel de la banque alimentaire ainsi : " [la banque alimentaire] est ouverte de $10 \mathrm{~h}$ à 13 h, mais [je] préférerais [qu'elle soit ouverte] de $9 \mathrm{~h}$ à $16 \mathrm{~h}$ ». Les autres obstacles étaient le fait de ne pas savoir à quels endroits les programmes d'aide alimentaire étaient offerts dans la ville, la qualité des aliments (décrits comme appartenant surtout à la catégorie de la " malbouffe »), l'obligation de montrer une preuve d'identité, le fait de ne pas pouvoir choisir ses aliments préférés et enfin le manque d'espace dans les locaux de la banque alimentaire rendant ces derniers inaccessibles aux personnes en fauteuil roulant ou accompagnées d'enfants en poussette. Quant à la qualité des aliments, une mère de deux enfants a raconté : « Lorsque je suis rentrée à la maison, j'ai réalisé qu'au moins la moitié [des denrées fournies par la banque alimentaire] étaient des gâteaux aux fruits, des beignets et des biscuits, et je me suis mise à pleurer... Je me suis demandée, comment je vais pouvoir nourrir mon enfant? »

\section{Solutions envisageables pour améliorer l'accès aux programmes d'aide alimentaire}

Trois répondants ont souhaité que les programmes d'aide alimentaire soient accessibles pendant un plus grand nombre de jours de la semaine et que les établissements soient ouverts le matin, l'après midi et le soir. Une mère de trois enfants a déclaré : " La plupart des programmes [sont] accessibles le lundi, le mercredi et le vendredi... [Ce serait] bien si quelque chose était offert les autres jours ». Plusieurs autres répondants ont évoqué le thème des réseaux de soutien social, par lesquels les gens peuvent s'entraider en cultivant des légumes et en les mettant en commun, ou en s'assurant qu'il reste assez de nourriture pour les autres. Une mère de deux enfants travaillant à temps partiel a décrit son système de banque alimentaire idéal : " Il faut être juste envers les autres ne pas être avide ou égoïste - ne pas en prendre trop, mais juste assez pour s'en tirer ». Enfin, treize répondants (37\%) ont déclaré ne voir que peu ou pas d'obstacle à l'accès aux programmes d'aide alimentaire.

\section{Obstacles à la consommation de légumes et de fruits}

Douze répondants (34\%) ont mentionné comme obstacle à la consommation de légumes et de fruits le fait de ne pas en avoir assez. Une mère d'un enfant travaillant à temps partiel a déclaré qu'elle n'avait pas besoin d'encouragements pour consommer davantage de légumes et de fruits, mais, a-t-elle déclaré, « [Je] n’en mange pas parce que je n'en ai pas [à la maison] ». Une mère de trois enfants monoparentale a expliqué qu'elle ne mange pas davantage de légumes ou de fruits parce qu'elle est en situation d'insécurité alimentaire : «Si je savais que j'en avais assez pour mes fils, j'en mangerais plus moimême ». Le fait de ne pas avoir assez d'argent pour acheter des fruits et des légumes et le prix élevé de ces denrées sont d'autres thèmes qui ont été fréquemment abordés. Lorsqu'on lui a demandé "Qu'est ce qui vous encouragerait à manger davantage de légumes et de fruits? », un homme d'âge moyen célibataire et sans emploi a répondu : « [Je] ne gagne pas assez d'argent. Si j'avais plus d'argent, c'est sûr que j'achèterais des fruits ». Une mère de deux enfants travaillant et seul soutien de sa famille a expliqué ce qui pourrait l'aider à consommer davantage de fruits et de légumes : «Avoir plus d'argent... ils disent [que les fruits et les légumes] ne sont pas chers, mais ce n'est pas vrai ». 


\section{Solutions envisageables pour augmenter la consommation de légumes et de fruits}

Onze répondants $(31 \%)$ ont suggéré que le programme d'aide alimentaire utilise ou propose un programme d'achat en grande quantité de fruits et légumes frais produits localement, comme "Bonne boîte bonne bouffe » ou la "Boîte verte » (Good Food Box), pour aider les gens à accroître leur consommation de ces denrées. Seize répondants (46\%) ont déclaré que la quantité consommée dépendait en partie du prix des fruits et légumes et de leur disponibilité dans les programmes d'aide alimentaire. Dix répondants (29 \%) ont mentionné l'adhésion à un programme de jardinage, ou le fait de disposer d'un jardin ou d'un espace plus grand pour faire pousser leurs fruits et légumes. Dix répondants (29\%) ont affirmé qu'ils consommeraient davantage de fruits et de légumes s'ils étaient mieux informés des avantages procurés par la consommation de ces aliments, s'ils avaient des recettes ou suivaient des cours de cuisine, et s'ils savaient comment conserver les fruits et les légumes pendant plus longtemps sans qu'ils se gâtent.

\section{Raisons de l'accès aux programmes d'aide alimentaire plus d'une fois par mois}

Sept répondants (20\%) ont expliqué qu'il leur fallait se rendre plusieurs fois par mois dans différentes banques alimentaires dans la grande région parce qu'aucun de ces établissements ne leur fournit une quantité de nourriture suffisante. Une mère de deux enfants travaillant à temps partiel a décrit son expérience dans une banque alimentaire ainsi : «On reçoit une boîte de soupe à la tomate pour deux semaines et un paquet de pâtes pour une famille de six... ce n'est pas assez! » Deux répondants ont souligné le manque de variété des denrées offertes par les banques alimentaires; une mère de deux enfants travaillant à plein temps a expliqué pourquoi elle fréquentait plusieurs fournisseurs d'aliments au cours du mois : "Ça ne me permet pas [autrement] d'avoir une alimentation équilibrée... Je ne peux pas avoir des aliments appartenant à chacun des quatre groupes en fréquentant les banques alimentaires ». Deux répondants ont expliqué que la fréquentation d'une seule une banque alimentaire limitait leur choix; une femme sans emploi mère de deux adolescents a déclaré : "[Une certaine banque alimentaire] décide pour moi : je n'ai pas besoin de champignons, de haricots et de tomates ». Quatorze répondants (40\%) ont déclaré qu'ils aimeraient pouvoir utiliser plusieurs programmes d'aide alimentaire chaque semaine.

\section{Avoir assez de nourriture, cela veut dire...}

Pour quatorze répondants (40\%), avoir assez de nourriture signifie être capable d'offrir à ses enfants une alimentation saine, nutritive et variée. Une mère célibataire de deux enfants qui travaille à temps partiel a expliqué ce que signifie pour elle avoir assez de nourriture : " [Cela] ne veut pas dire avoir le choix... mais avoir au moins un aliment de chaque groupe pour les enfants et pour moi à chaque repas - se contenter de ce qu'on a ". Pour d'autres, avoir assez de nourriture, c'est avoir l'assurance que "chaque membre de la famille a tout ce dont il a besoin ", ne pas avoir à s'inquiéter au sujet du budget ou ne pas craindre que les enfants aient faim. Pour une mère travaillant à temps partiel, avoir assez de nourriture, c'est « savoir qu'il y a assez à manger dans le réfrigérateur ou dans le garde-manger jusqu'à la réception de mon prochain chèque ». De l'avis de cinq répondants (14\%), avoir une quantité adéquate de nourriture, c'est être capable de manger plusieurs fois par jour ou laisser les enfants manger autant qu'ils le souhaitent. Sept répondants (20\%) ont également déclaré souhaiter avoir régulièrement une alimentation saine et "nourrir chaque jour toute leur famille ».

\section{Discussion}

Dans cette évaluation des besoins, nous avons constaté que 1) les problèmes de transport, la quantité d'aliments offerte et les horaires des programmes alimentaires limitaient l'accès à la nourriture; 2) la disponibilité des fruits et légumes et le revenu limitaient la consommation de ces denrées chez les personnes en situation d'insécurité alimentaire; 3) pour des raisons de quantité et de qualité des aliments, les répondants devaient se tourner vers les programmes d'aide alimentaire plus d'une fois par mois; 4) pour la majorité des répondants, avoir assez de nourriture, c'était être en mesure d'offrir à ses enfants des aliments nutritifs en quantité suffisante.
Dans plusieurs études visant à évaluer les programmes caritatifs d'aide alimentaire, on a observé que les bénéficiaires recevaient souvent des quantités de nourriture insuffisantes et que la qualité de celle-ci laissait à désirer ${ }^{29-31}$. Teron et Tarasuk ont évalué 85 paniers de nourriture reçus par des clients de la Toronto Daily Food Bank et ont observé que plus de la moitié des ménages composés de trois personnes ou plus recevaient une quantité d'aliments équivalant à moins de trois jours de consommation ${ }^{29}$. De surcroît, plus de $78 \%$ de ces paniers contenaient au moins un article alimentaire endommagé ou périmé29. Selon Hamelin et collab., pour les ménages à faible revenu en situation d'insécurité alimentaire, satisfaire ses besoins physiques de base en consommant une quantité suffisante de nourriture a la même importance que d'avoir une alimentation diversifiée et équilibrée ${ }^{32,33}$. Dans notre évaluation des besoins, les répondants ont eux aussi souligné l'importance de satisfaire leurs besoins physiques de base en quantité et en qualité de nourriture; la non satisfaction de ces besoins pourrait être une des raisons pour lesquelles près de la moitié des répondants souhaitaient pouvoir profiter des services et des programmes d'aide alimentaire plus souvent, c'est-à-dire une fois par semaine.

Dans notre évaluation des besoins, la plupart des répondants étaient des mères. D’autres études canadiennes ont également fait ressortir l'importance vitale pour les mères de faire en sorte que leurs enfants bénéficient d'une nutrition optimale ${ }^{33-36}$. Pour elles, avoir assez de nourriture, c'était s'acquitter de l'obligation de subvenir aux besoins de leurs enfants. Ces mères ne ménagent pas leurs efforts pour combler la faim de leurs enfants et n'hésitent pas à fréquenter les programmes d'aide alimentaire plusieurs fois par mois, en dépit de la stigmatisation associée au recours aux banques alimentaires et du sentiment de perte de dignité qu'il suscite ${ }^{33,37}$.

Comme les banques alimentaires et les programmes similaires sont fortement tributaires de la charité et des dons de produits, il n'y a aucune garantie concernant le niveau des stocks ou le type d'aliment distribué en un lieu ou à un moment donné. Il ne s'agit donc pas d'une source d'approvisionnement en 
nourriture fiable pour les personnes en situation d'insécurité alimentaire tributaires uniquement de ces programmes pour nourrir leur famille au quotidien ${ }^{22,29,32,33}$.

Les répondants ont été nombreux à affirmer que le transport était pour eux un obstacle à l'utilisation des programmes d'aide alimentaire. Cette constatation n'est pas le seul fait des personnes à faible revenu, car dans une étude menée récemment auprès de tous les résidents du Comté de Northumberland, le transport était l'une des trois principales préoccupations soulevées ${ }^{38}$. Pour les personnes à faible revenu qui ont à peine assez d'argent pour les besoins de la vie quotidienne, le transport en commun est un luxe ${ }^{39}$ et pour les rares personnes qui ont les moyens d'avoir un véhicule, celui-ci est rarement en état de fonctionner et fiable ${ }^{40}$. D'après notre étude des besoins, la marche à pied est le moyen de transport le plus courant ou privilégié parce qu'il n'est pas coûteux; bien sûr, le fait de devoir porter les boîtes de nourriture rend le retour problématique. Dans une étude sur le vécu de mères à faible revenu ayant des enfants à charge, Bostock a constaté que 82 $\%$ des répondantes ne possédaient pas de voiture et devaient marcher pour se déplacer ${ }^{41}$ et que, même si elles trouvaient stressant et physiquement fatigant de marcher, elles étaient limitées aux ressources accessibles à pied. En résumé, l'absence de moyen de transport restreint le mode de vie d'un individu et son accès aux ressources, dont la quantité de nourriture qu'il peut rapporter chez lui.

Dans notre évaluation des besoins, de nombreux répondants ont déclaré qu'ils consommeraient davantage de fruits et de légumes si des produits frais de ce type étaient disponibles chez eux. Un échange plus approfondi a révélé que la raison première de l'insuffisance des fruits et des légumes à la maison était que les produits frais n'étaient pas abordables et étaient rarement offerts par les programmes d'aide alimentaire. Des études ont montré que les individus dont les conditions socio économiques sont les plus défavorables tendent à consommer moins de fruits et de légumes que ceux qui jouissent d'un statut plus enviable à cet égard ${ }^{42-48}$. Santé Canada recommande que les adultes âgés de 19 à 50 ans consomment chaque jour au moins sept portions de fruits et de légumes $^{49}$. Toutefois, pour les personnes dont les ressources sont limitées, le prix des fruits et des légumes rend souvent impossible la consommation du nombre de portions recommandé. Le revenu, tout comme les autres déterminants sociaux tels que l'emploi, le logement, l'éducation et l'accès aux services, a des conséquences profondes sur la santé des individus et des communautés ${ }^{50-52}$, certains allant même jusqu'à dire qu'il s'agit du plus important ${ }^{53,54}$.

Si nos observations vont dans le même sens que celles des autres publications sur les obstacles à l'accès à la nourriture, notre étude présente cependant quelques limites. Tout d'abord, pour intimider le moins possible les répondants et favoriser ainsi leur participation, les entrevues n'ont pas été enregistrées. Les intervieweurs jouaient deux rôles simultanément, mener l'entrevue tout en consignant les réponses. Dans ces conditions, ils ont pu obtenir des renseignements importants mais oublier de les consigner intégralement, ou encore ils ont pu prendre des notes avec diligence mais oublier de demander des éclaircissements lorsque c'était nécessaire. Il faut également compter avec la possibilité d'un biais lié à l'intervieweur, puisque celui-ci doit filtrer rapidement les réponses pour noter les mots-clés, les expressions ou les phrases de chaque répondant. Les données passent ainsi par un niveau préliminaire de tri et d'analyse qui peut varier légèrement d'un intervieweur à l'autre; de plus, il est difficile d'évaluer dans quelle mesure l'information a été filtrée par ce processus.

Deuxièmement, un nombre plus élevé d'entrevues a été effectué à l'un des deux centres choisis. Par conséquent, la population globale de l'échantillon pourrait ne pas être représentative de l'ensemble des individus en situation d'insécurité alimentaire.

En dépit de ces limites, notre évaluation des besoins a mis en lumière la nécessité d'approfondir les questions suivantes :

1) Disponibilité des denrées : prendre des mesures pour que certains types de denrées soient offerts par les programmes d'aide alimentaire et que les quantités d'aliments distribuées soient adaptées à la taille du ménage.
2) Transport : prendre des mesures pour offrir un moyen de transport en commun abordable permettant aux gens de se rendre dans les banques alimentaires et d'en revenir.

3) Réseaux de soutien : prendre des mesures pour accroître les possibilités de réseautage social afin de permettre aux gens de s'entraider.

4) Programme d'achat en grande quantité de fruits et légumes frais produits localement : prendre des mesures pour que le programme soit abordable, en particulier pour les familles et les personnes à faible revenu.

5) Utilisation de jardins communautaires : prendre des mesures pour susciter de l'intérêt pour les jardins communautaires et favoriser l'acquisition de compétences à cet égard afin d'accroître la consommation de fruits et de légumes.

Pour résoudre ces problèmes d'accès à la nourriture, il faudra relever de nouveaux défis. Par exemple, il est crucial que tous les intervenants, y compris les partenaires communautaires et les communautés locales, unissent leurs efforts pour surmonter les obstacles.

Les programmes de banque alimentaire et de soupe populaire n'ont jamais eu comme vocation d'être des services à long terme. Ils visaient à l'origine à soulager temporairement les personnes aux prises avec des difficultés économiques, afin de leur permettre de consacrer leur revenu au paiement de leurs factures et à leurs autres besoins quotidiens. Ces programmes sont devenus permanents, et ne pourront être supprimés que si l'on s'attaque en parallèle à d'autres facteurs socio-économiques tels que le transport, l'emploi, l'éducation, la garde des enfants et l'accès au logement. Si la route est encore longue avant de pouvoir éradiquer ces problèmes sociaux, chaque individu peut, dans l'intervalle, contribuer à faire tomber les obstacles et à réduire les facteurs de risque de maladies chroniques en s'attaquant d'abord à l'accès aux denrées alimentaires. Cette recherche contribuera à éclairer la prise de décisions au niveau local et à améliorer l'offre de programmes dans le domaine de la sécurité alimentaire. 


\section{Remerciements}

Nous souhaitons remercier le Réseau de prévention et de dépistage du cancer, Région du Grand Toronto, de la Société canadienne du cancer pour l'appui financier qu'il a apporté à la présente recherche. Nous sommes reconnaissants aux deux organisations non gouvernementales au sein desquelles nous avons recruté les répondants pour l'aide qu'elles nous ont apportée en matière de collecte des données et de recrutement. Nous exprimons notre gratitude aux participants qui nous ont fait part de leur expérience et de leur vécu. Nos remerciements vont également à Lesley Hamilton, directrice administrative de Literacy Ontario Central South, et à Sasha Korper, consultante en alphabétisme chez les jeunes enfants, pour leur aide au sujet de la lisibilité et du niveau d'alphabétisation de notre lettre d'information aux participants. Enfin, nous remercions Gayle Broad, professeure adjointe à l'Université Algoma, Lynn Scruby, professeure adjointe à la Faculté des sciences infirmières de l'Université du Manitoba et Valerie Tarasuk, professeure à la Dalla Lana School of Public Health de l'Université de Toronto, pour leurs commentaires sur la méthodologie de recherche préliminaire.

\section{Références}

1. Gordon D, Shaw M, Dorling D, Davey Smith $\mathrm{G}$, editors. Inequalities in health: the evidence presented to the independent inquiry into inequalities in health. Bristol (UK): Policy Press; 1999. (Studies in poverty, inequality and social exclusion.)

2. Sram I, Ashton J. Millennium report to Sir Edwin Chadwick. Br Med J. 1998;317:592 6.

3. Pantazis C, Gordon D. Tackling inequalities: Where are we now and what can be done? Bristol (UK): Policy Press; 2000. (Studies in poverty, inequality and social exclusion.)

4. Organisation mondiale de la Santé. Prévention des maladies chroniques : un investissement vital [Internet]. Genève $(\mathrm{CH})$ : Organisation mondiale de la Santé; 2006 [consulté le 27 janv. 2010]. Disponible à : http://www .who.int/chp/chronic_disease_report/part1 /fr/index.html
5. Lynch J, Smith GD, Hillemeier M, Shaw M, Raghunathan T, Kaplan G. Income inequality, the psychosocial environment, and health: comparisons of wealthy nations. Lancet. 2001;358(9277):194-200.

6. Wilkins R, Berthelot JM, Ng E. Tendances de la mortalité selon le revenu du quartier dans les régions urbaines du Canada, 1971-1996. Rapp. santé. 2002;13 Suppl:1-28.

7. Gwatkin DR. Inégalité de santé et santé des pauvres : que sait-on et que peut-on faire? Bull. Organ. mond. Santé. 2000;78(1):3-18.

8. Williamson DL. The role of the health sector in addressing poverty. Can J Public Health. 2001;92(3):178-83.

9. Gyorfi-Dyke E. Poverty and chronic disease: recommendations for action [Internet]. Ottawa (Ont.): Chronic Disease Prevention Alliance of Canada; 2008 [consulté le 27 janv. 2010]. PDF téléchargeable à partir du lien : http://www.healthyenvironmentforkids.ca /sites/healthyenvironmentforkids.ca/files /cpche-resources/CDandPoverty_CDPAC.pdf

10. Archeson D. Independent inquiry into inequalities in health [Internet]. United Kingdom: Her Majesty Stationary Office; 1998 [consulté le 9 déc. 2009]. Consultable à la page : http://www.archive.official-documents.co.uk /document/doh/ih/ih.htm

11. Lightman E, Mitchell A, Wilson B. Poverty is making us sick: a comprehensive survey of income and health in Canada. Toronto (Ont.): Wellesley Institute; 2008.

12. Wilson B. Sick and tired: the compromised health of social assistance recipients and the working poor in Ontario. Toronto (Ont.): The Community Social Planning Council of Toronto (CSPC-T); 2009.

13. Lightman E, Herd D, Mitchell A. Precarious lives: work, health and hunger among welfare recipients in Toronto. J Policy Prac. 2008;7(4):242-59.

14. Vozoris N, Davis B, Tarasuk V. The affordability of a nutritious diet for households on welfare in Toronto. Can J Public Health. 2002;93(1):36-41.

15. Riches G. Food banks and the welfare crisis. Ottawa (Ont.): Lorimer; 1986.
16. Kirkpatrick SI, Tarasuk V. Adequacy of food spending is related to housing expenditures among lower income Canadian households. Public Health Nutr. 2007;10(12);1464-73.

17. Williams PL, Johnson CP, Kratzmann ML, Johnson CS, Anderson BJ, Chenhall C. Can households earning minimum wage in Nova Scotia afford a nutritious diet? Can J Public Health. 2006;97(6):430-4.

18. Tarasuk V, McIntyre L, Li J. Low income women's dietary intakes are sensitive to the depletion of household resources in one month. J Nutr. 2007;137:1980-7.

19. Kirkpatrick SI, Tarasuk V. Food insecurity is associated with nutrient inadequacies among Canadian adults and adolescents. J Nutr. 2008;138:604-12.

20. Agriculture et Agroalimentaire Canada. Plan d'action du Canada pour la sécurité alimentaire [Internet]. Ottawa (Ont.): Agriculture et Agroalimentaire Canada; 1998 [consulté le 29 oct. 2009]. Disponible à : http://www .agr.gc.ca/misb/fsec-seca/pdf/action_f.pdf

21. Dietitians of Canada. Community food security: position of Dietitians of Canada [Internet]. Toronto: Dietitians of Canada; 2007 [consulté le 9 avr. 2009]. PDF téléchargeable à partir de la page : http://www.dietitians.ca/DietitiansView/Community-Food-Security.aspx

22. Dietitians of Canada. Individual and household food insecurity in Canada: position of Dietitians of Canada [Internet]. Toronto: Dietitians of Canada; 2005 [consulté le 9 avr. 2009]. PDF téléchargeable à partir de la page : http:// www.dietitians.ca/Dietitians-View/Individualand-Household-Food- Insecurity.aspx

23. Vogt J, Tarasuk V. Analysis of Ontario sample in cycle 2.2 of the Canadian Community Health Survey, 2004 [Internet]. Ontario: Public Health Research, Education and Development; 2007 [consulté le 8 janv. 2009]. Consultable à la page : http://www.phred-redsp.on.ca/CCHSReport.htm

24. Statistique Canada. Profils des communautés de 2006 : division de recensement [Internet]. Ottawa (Ont.): Statistique Canada; 2009 [consulté le 3 déc. 2009]. Consultable à la page : http:// www12.statcan.ca/census-recensement/2006 /dp-pd/prof/92-591/index.cfm?Lang = F 
25. Lacey A, Luff D. Qualitative data analysis [Internet]. Nottingham (UK): The NIHR Research Design Service for the East Midlands; 2007 [mis à jour en 2009; consulté le 29 oct. 2009]. PDF téléchargeable à partir de la page : http://www.rds-eastmidlands .nihr.ac.uk/resources/cat_view/13-resourcepacks.html?start $=5$

26. Srivastava A, Thomson SB. Framework analysis: a qualitative methodology for applied policy research. JOAAG. 2009;4(2):72-9.

27. La Pelle N. Simplifying qualitative data analysis using general purpose software tools. Field Method. 2004;16(1):85-108.

28. Ryan GW. Using a word processor to tag and retrieve blocks of text. Field Method. 2004;16(1):109-30.

29. Teron AC, Tarasuk V. Charitable food assistance: what are food bank users receiving? Can J Public Health. 1999;90(6):382-4.

30. Irwin JD, Ng VK, Rush TJ, Nguyen C, He M. Can food banks sustain nutrient requirements? A case study in Southwestern Ontario. Can J Public Health. 2007;98(1):17-20.

31. Tse C, Tarasuk V. Nutritional assessment of charitable meal programmes serving homeless people in Toronto. Public Health Nutr. 2008;11(12):1296-305

32. Hamelin AM, Mercier C, Bedard A. Discrepancies in households and other stakeholders viewpoints on the food security experience: a gap to address. Health Educ Res. 2010;25(3):401-2.

33. Hamelin A-M, Beaudry $M$, Habicht J-P. Characterization of household food insecurity in Québec: food and feelings. Soc Sci Med. 2002;54:119-32.

34. McIntyre L, Glanville NT, Raine KD, Dayle JB, Anderson B, Battaglia N. Do low-income lone mothers compromise their nutrition to feed their children? CMAJ 2003;168:686-91.

35. McIntyre L, Glanville NT, Officer S, Anderson B, Raine KD, Dayle JB. Food insecurity of low-income lone mothers and their children in Atlantic Canada. Can J Public Health. 2002; 93(6):411-5.
36. Tarasuk V, Maclean H. The food problems of low income single mothers: an ethnographic study. Can Home Econ J. 1990;40:76-82.

37. Riches G. Food banks and food security: welfare reform, human rights and social policy. Lessons from Canada? Soc Policy Admin. 2002;36(6):648-63.

38. Northumberland United Way. Community matters: community consultation final report [Internet]. Cobourg (Ont.); 2006 [consulté le 25 août 2010]. PDF téléchargeable à partir du lien : http://www.cobourg.unitedway.ca/Local _images/cobourg/CommunityMatters.pdf

39. Northumberland Poverty Reduction Action Committee. Report from the Northumberland Poverty Reduction Action Committee community action day. Cobourg (Ont.): Northumberland Poverty Reduction Action Committee; 2008.

40. Garasky S, Fletcher CN, Jensen HH. Transiting to work: the role of private transportation for low income households. J Consum Aff. 2006;40(1):64-89.

41. Bostock L. Pathways of disadvantage? Walking as a mode of transportation among low-income mothers. Health Soc Care Community. 2000;9(1):11-8.

42. Perez CE. Consommation de fruits et de légumes. Rapp. santé. 2002;13(3):25-34.

43. Subar AF, Heimendinger J, Patterson BH. Fruit and vegetable intake in the United States: the baseline survey of the Five A Day for Better Health Program. Am J Health Promot. 1995;9(5):352-60.

44. Xie B, Gilliland FD, Li YF, Rockett HRH. Effects of ethnicity, family income, and education on dietary intake among adolescents. Prev Med. 2003;36:30-40.

45. Giskes K, Turrell G, Patterson C, Newman B. Socioeconomic differences among Australian adults in consumption of fruit and vegetables and intakes of vitamins $\mathrm{A}, \mathrm{C}$ and folate. J Hum Nutr Diet. 2002;15:375-85.

46. Mishra G, Ball K, Arbuckle J, Crawford D. Dietary patterns of Australian adults and their association with socioeconomic status: results from the 1995 National Nutrition Survey. Eur J Clin Nutr. 2002;56:687-93.

47. Reicks M, Randall RL, Haynes BJ. Factors affecting consumption of fruits and vegetables by low-income families. J Am Diet Assoc. 1994;94(11):1309-11.

48. Kirkpatrick S, Tarasuk V. The relationship between low income and household food expenditure patterns in Canada. Public Health Nutr. 2003;6(6):589-97.

49. Santé Canada. Bien manger avec le Guide alimentaire canadien [Internet]. Ottawa (Ont.): Santé Canada; 2009 [consulté le 7 janv. 2009]. Consultable à la page : http://www.hc-sc.gc .ca/fn-an/food-guide-aliment/index-fra.php

50. Wilkinson R, Marmot M, directeurs. Les déterminants sociaux de la santé : les faits. $2^{\mathrm{e}}$ éd. [Internet]. Copenhague (DK): Organisation mondiale de la santé; 2004 [consulté le 3 déc. 2009]. PDF téléchargeable à partir du lien : http://www.euro.who.int/_data/assets/pdf_file /0006/98439/E82519.pdf

51. Raphael D. Health effects of economic inequality. Can Rev Soc Policy. 1999;44:25-40.

52. Raphael D. Health inequalities in Canada: current discourses and implications for public health action. Crit Public Health. 2000;10:193-216

53. Raphael D. Poverty, income inequality, and health in Canada [Internet]. Toronto (ON): The CSJ Foundation for Research and Education; 2002 [consulté le 3 déc. 2009]. PDF téléchargeable à partir du lien : http://www .socialjustice.org/uploads/pubs/PovertyIncomeInequalityandHealthinCanada.pdf

54. Commission des déterminants sociaux de la santé. Combler le fossé en une génération : instaurer l'équité en santé en agissant sur les déterminants sociaux : rapport final de la Commission des Déterminants sociaux de la santé. Genève $(\mathrm{CH})$ : Organisation mondiale de la santé; 2009. PDF téléchargeable à partir de la page : http://www.who.int/social_determinants/thecommission/finalreport/fr/index.html 